Investigaciones Fenomenológicas, vol. Monográfico 5, 2015, 345-356. e-ISSN: $1885-1088$

\title{
LA IDEA TRASCENDENTAL DEL HOMBRE: REFLEXIONES ANTROPOLÓGICAS DESDE IDEAS DE 1913
}

The Transcendental IDEA OF MAN:

ANTHROPOLOGICAL REFLECTIONS FROM IDEAS OF 1913

\author{
Marcela Venebra \\ FFyL-UNAM / FH-UAEM \\ México \\ marvenebra@gmail.com
}

Resumen: Este artículo explora tanto las dificultades como las posibilidades de la idea de una antropología filosófica de base o fundamento fenomenológico. Toma como punto de partida los aspectos críticos que, desde un punto de vista externo a la propia fenomenología, se oponen a la posibilidad u opacan la necesidad trascendental de la antropología filosófica; y se propone como meta la exposición de aquellos elementos que, desde el punto de vista antropológico, son fundamentales para comprender las motivaciones de la emergencia de este 'problema antropológico' en el contexto de la fenomenología trascendental.

Palabras clave: antropología filosófica, fenomenología trascendental.

\begin{abstract}
LThis paper explores the problems as well as the feasability about the idea of a philosophical anthropology with a phenomenological basis. Its starting point is the critical issues that, from an external point of view to phenomenology itself, preclude the possibility or transcendental necessity of a philosophical anthropology. The objective is to disclose those elements that from an anthropological point of view are fundamental to understanding the motives of the emergence of this 'anthropological problem' within the context of transcendental phenomenology
\end{abstract}

Key Words: Philosophical Anthropology, Transcendental Phenomenology.

El objetivo principal de estas páginas es -apenas- establecer algunas de las consideraciones que exige la siguiente tesis: sin la dimensión trascendental del hombre, ninguna antropología (ni cultural ni filosófica) es posible; decir que sin trascendentalidad no hay antropología es, al mismo tiempo, afirmar que sólo la trascendentalidad 'salva al hombre', pues ¿qué queda del hombre sin la dimen- 
sión trascendental de su ser? ¿No es la fenomenología trascendental una ganancia espiritual humana? ${ }^{1}$ Quisiera clarificar las motivaciones de estos cuestionamientos a través de tres momentos en los que sólo apuntaré las direcciones que, en el curso de la obra Husserl, nos conducen a esto que también podría llamarse: el tema o problema antropológico de la fenomenología trascendental. En un primer momento abordaré a manera de marco crítico algunos de los argumentos de Hans Blumenberg (por ser también los más corrientes y los de más peso) en contra de la posibilidad de una antropología fenomenológica que mantenga el sentido trascendental de la filosofía husserliana. Frente a esto que el propio Blumenberg llama, la «carencia antropológica de la fenomenología»² plantearé, en un segundo momento, la limitación de la idea de hombre con la que opera Husserl y que aparece, de modo implícito, en la crítica husserliana del escepticismo que motiva la puesta en marcha de la epojé. Finalmente, y frente a estas dos perspectivas «de la falta» (de la falta antropológica de la fenomenología, y del hombre en falta), planteo la posibilidad de una idea trascendental de lo humano a partir de la consideración trascendental del cuerpo que se deja extraer del segundo libro de las Ideas y, concretamente, de los análisis de las ubiestesias respecto de la génesis de la identidad yoica, es decir, tanto del yo personal, como del yo trascendental. En realidad este es un tema definitorio del problema antropológico, y ya Javier San Martín lo ha tratado con detalle en diversos momentos ${ }^{3}$, tampoco es la única salida antropológica de la fenomenología quizás, sólo, la más inmediata en la obra accesible de Husserl y, dada la magnitud y el espesor del problema, sólo me dará tiempo aquí de trazar algunos apuntes.

\section{LA CARENCIA ANTROPOLÓGICA DE LA FENOMENOLOGÍA}

¿Cómo pretender una reforma de sentido antropológico desde una filosofía que debe prescindir de lo humano como toda filosofía, quizás, lo hace o debería hacerlo? ¿Cuál es el sentido de esta renuncia decisiva a la certeza del mundo?,

\footnotetext{
${ }^{1}$ La filosofía es aquí comprendida como "Aquel momento de la vida en que con la génesis de una razón de verdad se gesta un hombre nuevo." Nicol, E., La reforma de la filosofía, F.C.E., México, 1989, p. 29. 2 Blumenberg, H., Descripción del ser humano, F.C.E., Buenos Aires, 2011, p. 32. Trad. Griselda Mársico. (En adelante: $D S H$ ).

3 Sobre la relación entre antropología y fenomenología Javier San Martín ha escrito diversos trabajos desde hace más de dos décadas. Baste aquí citar alguno de los textos más sintéticos de este esfuerzo: Fenomenología y antropología, Lectour-UNED, Buenos Aires/Madrid, 2005.
} 
¿cómo es que este primer movimiento: dejar de saber lo humanamente sabido, dejar de saberse «hombre» para penetrar en la vida, termina por fundar (o refundar o re-formar) la vocación antropológica?, ¿tal cosa es probable? ¿La antropología filosófica no es acaso la declarada humillación de toda aspiración auténticamente científica? El problema de la antropología filosófica es que parece ella misma un hierro de madera. Formulado de otro modo, se diría -como lo hace Blumenberg, por ejemplo- que la carencia antropológica de la fenomenología sólo se suple con la declaratoria del «fracaso» trascendental. La idea del hombre que se niega la fenomenología husserliana es la misma que arruina el proyecto filosófico universal. Tal derrota, si estuviéramos dispuestos a asumirla, se origina en la brecha que abre la epojé fenomenológica entre el yo puro y el yo humano. El yo puro es el título de una conciencia universal ilocalizada, no corporal y no humana.

Sin embargo, y antes de resignarse a ningún «fracaso», término que en filosofía resulta al menos cuestionable, quisiera hacer una pregunta previa a la infranqueable oposición trascendental/humanidad-mundanidad: ¿qué es la idea del hombre antes de la reducción fenomenológica? ¿Qué es el hombre, incluso el hombre que filosofa (y que es aquí el que con mayor razón nos interesa) antes de llevar a cabo la reducción trascendental? ¿Qué es, en fin, la antropología, la idea del hombre antes de ser filosóficamente pensada; filosóficamente, es decir, desde una filosofía pura? La no coincidencia entre el yo puro y el yo humano que revela la epojé, ¿no exige también una comprensión de lo que representa esta escisión de la autopercepción?

La epojé y el yo puro conseguido mediante ella son los principales obstáculos que impiden formular una antropología no limitada a una ontología regional $y$, sin embargo, bajo una nueva dimensión crítica, la exigida en sentido estricto para una filosofía de la filosofía (la que se gana después de la reducción), son también las vías de acceso al tema del hombre en el seno de la fenomenología sólo como trascendental. El yo puro gana una nueva y más concreta dimensión a través de la corporalidad sintiente, que opera como génesis de su identidad. Pero esta 'ganancia', como se verá, no es sólo ganancia del yo puro, lo es también del yo humano, que, en tanto concreción, sólo se hace visible respecto de la primera idea -aun implícita- del hombre que rechaza Husserl como fundamento en su crítica del escepticismo. 


\section{LA ANTROPOLOGÍA DE LA CARENCIA Y LA CRÍTICA AL ESCEPTICISMO}

En las Investigaciones lógicas Husserl advierte el tránsito que todo relativismo ofrece al escepticismo. De cara al relativismo hay que decir que no puede haber una 'verdad para', pues "lo verdadero es absolutamente verdadero, no importa si son ángeles o dioses" ${ }^{\prime 4}$ los que enuncian la verdad. Aquí Husserl descarta lo humano como fundamento o determinación de las leyes lógicas, esto es, la humanidad en tanto especie biológicamente determinada, pues "la constitución de una especie es un hecho y de hechos sólo pueden sacarse hechos" ${ }^{\prime 5}$. Decir que hay una verdad «para la especie humana» es negar la verdad. Es destacable el hecho de que en esta crítica del relativismo se reafirme en cierto modo una idea de lo humano como pura determinación psicofísica, esa idea del hombre que las ciencias de la naturaleza han establecido con toda la fuerza del dogmatismo ${ }^{6}$ ingenuo que caracteriza al positivismo. Ya en las Ideen esta idea, que es la misma idea del hombre que ha de quedar puesta entre paréntesis, es tanto más rica que trece años antes. El hombre es sujeto personal mundano, existente en un mundo espiritual, estructurado valorativa, cultural e históricamente. El hombre ya no es sólo un objeto de la ciencia de la naturaleza, sino también un objeto de las ciencias del espíritu. Es un objeto por así decirlo, espiritualizado, pero en tanto objeto, es decir, en tanto trascendencia no inscripta en la inmanencia intencional debe quedar al igual que todo lo que éste supone, su mundo, su historia, sus congéneres, en el paréntesis de la epojé. Acaso la investigación nos muestre en algún momento la forma en que el yo puro que la epojé descubre se objetiva como yo humano, pero esto no implica ninguna necesidad de hecho.

\footnotetext{
${ }^{4}$ Husserl, E. Investigaciones lógicas, 1., Alianza, Madrid, 2006, §36., p. 114. Trad. Manuel García Morente y José Gaos. (En adelante $I L$ )

${ }^{5}$ Husserl, E., IL, p.115.

${ }^{6}$ Uso aquí el término dogmático en el sentido en que lo utiliza Husserl en el $\S 62$ de las Ideas. Aquí la actitud dogmática se opone a la crítica, como crítica de los supuestos y el sentido de las ciencias y del hace científico. La ingenuidad y el dogmatismo convergen en la forma en la que el científico tiene dados sus objetos, como ya dados y dados sobre un mundo cuyo sentido de ser se asume de por sí. Esta crítica es tarea la tarea que asume la fenomenología: "Pues a partir de fuentes esenciales puede verse intelectivamente que las ciencias involucradas son realmente (...) aquellas que han menester de la 'crítica', y de una crítica que ellas mismas no pueden por principio ejercer." Husserl, E., Ideas para una fenomenología pura y una filosofía fenomenológica. Libro primero: Introducción general a la fenomenología pura, F.C.E., México, 2013. § 62, p.118. Trad. José Gaos. Edición y refundición integral, Antonio Zirión Quijano. (En adelante Ideas $I$ )
} 
En 1931, en la conferencia sobre antropología y fenomenología que dicta Husserl en Berlín, ante a un auditorio inusitado para un filósofo capaz de convocar a mil seiscientos oyentes atentos al pronunciamiento definitivo de Husserl frente a Scheler, frente a Heidegger, frente a la mayor amenaza que le había surgido hasta entonces a su filosofía, "una antropología filosófica apoyada en la fenomenología", 7 pone Husserl en marcha otra idea del hombre que no es ya solamente- la del antropologismo criticado en las Investigaciones lógicas, ni exactamente- la del mundo entorno y la realidad personal expuesta en las Ideas, esta idea del hombre que Husserl ataca con tanto encono, es la idea historicista de lo humano, la que él supone en la base del existencialismo, que nombra una nueva dimensión no considerada por el naturalismo, aunque en cierta medida sigue la misma lógica determinista de las corrientes positivistas, porque al situarse críticamente frente a una idea más o menos desvirtuada del racionalismo, la afirma sin tocar sus fundamentos. Si antes el hombre era el producto de las determinaciones de su constitución psicofisiológica, ahora es el centro de confluencia de su circunstancia histórico-material. De cualquier manera, y ya de uno u otro lado de las posiciones propiamente antropológicas, tenemos una idea fallida del hombre, no autosuficiente. Tanto desde el naturalismo, como desde el historicismo, el hombre es la imposibilidad de su autonomía, es incapaz de dar cuenta de sí mismo por sí mismo, remitiendo siempre a una fuente externa de su propio sentido. Finalmente, el rechazo husserliano de la antropología (del antropologismo) está fundado en esta idea del hombre en falta, y esta es la motivación de la crítica del antropologismo. Este es el verdadero problema de la crítica husserliana, que yo no puedo confiar la meta de la ciencia estricta a un ser objeto -entre otros- que sólo puede explicarse por otra cosa, que no es absoluto sino carencia (falta de su propio sentido), que no es absoluto sino histórico, temporal, corporal y, sin embargo, hay un principio absoluto en el hombre que es la racionalidad, la idea de lo humano como ser para la verdad, y la racionalidad de la vida sólo puede asegurarse a través de una filosofía como ciencia estricta.

La misma idea que moviliza la crítica del escepticismo, la del hombre como ser de meros hechos, es la que Husserl se propone reformar a través de la filosofía de La crisis. Si bien, como nos dijo en las Investigaciones lógicas, la cons-

\footnotetext{
7 Blumenberg, H., DSH, p. 17.
} 
titución de una especie es un asunto fáctico que no puede servir como fundamento de la filosofía o la lógica, también es cierto que las ciencias de hechos no tienen la última palabra sobre el sentido de lo humano, y la filosofía surge con toda la fuerza de su necesidad frente al reduccionismo relativista que pone en crisis a la humanidad en tanto racional, en tanto filosófica o críticamente pensada.

La epojé tiene sentido como prevención de una metábasis ${ }^{8}$ motivada por esta idea naturalista de lo humano. El traspaso de los límites de lo fundante hacia lo fundado sólo es consecuencia de esta idea manca del hombre, de la idea del hombre como sobredeterminado, como entidad absolutamente explicable por las ciencias de la naturaleza, desde donde también puede explicarse, claro, en este sentido escéptico, el sentido y origen de la filosofía como obra de la especie humana. Pero una vez que la dimensión trascendental de esa humanidad (antes estrecha) aparece, el propio método fenomenológico ( $y$ esta es una meta recurrente en las Ideas) deberá ir ajustándose a los nuevos descubrimientos. ¿Cuándo es posible la crítica de la experiencia trascendental? ¿Cuándo el fenomenólogo puede volver sobre su operar y dar cuenta de la vida como el suelo de su quehacer? ¿Cuándo la fenomenología se vuelve filosofía de la filosofía? ¿No es aquí cuando reaparece la idea del hombre, sobre todo la del sujeto filósofo, el «yo fenomenólogo que medita» y que puede, por así decirlo, detener el curso de la vida corriente, detenerse a «otear el horizonte», llevar a cabo la epojé? Estos cuestionamientos nos conducen a una básica distinción entre fenomenología descriptiva y fenomenología crítica, filosofía como crítica de la experiencia trascendental, meta anunciada por Husserl en diversos momentos (las lecciones de $1910-11^{9}$ sobre los problemas fundamentales de la

\footnotetext{
${ }^{8}$ En el $\S 61$ de las Ideas I Husserl plantea como meta decisiva de la epojé, la evitación de una metábasis allos genos. La epojé tiene que ser el comienzo y una de las más potentes defensas frente al escepticismo, porque finalmente, el psicologismo con cuya crítica da inicio la crítica fenomenológica, no es sino la transgresión de los límites adecuados a una ciencia de hechos que pretende situarse como fundamento de todo conocimiento. "Tampoco sería necesario esmerarse en separar los distintos pasos, si no hubiese constantes tentaciones a incurrir en erróneas metábasis, especialmente en la interpretación de las objetividades de las disciplinas eidéticas. Son tentaciones tan fuertes, que amenazan incluso a quien se ha librado de las malas interpretaciones generales en dominios singulares." Husserl, E., Ideas I, § 61, P. 216.

9 "Aquí entra, [en el trascender de la fenomenología más allá del ámbito de lo absolutamente dado], en primer lugar, la idea de una crítica apodíctica de la experiencia fenomenológica a partir de sus formas fundamentales: percepción, retención, rememoración, etc., en el fenómeno (...) Primero se tiene que ganar en general la experiencia fenomenológica pura; sólo después puede ejercerse la crítica apodíctica." Husserl, E., Problemas fundamentales de la fenomenología, Alianza Universidad, Madrid, 1994, p. 95. Trad. Javier San Martín y César Moreno. Este anuncio programático anclado a la reducción de la evidencia fenomenológica a su apodicticidad aparece en distintos momentos tardíos y tempranos de la obra de Husserl, su anuncio en las Meditaciones cartesianas es uno de los más conocidos: "Tan sólo, en
} 
fenomenología y la Quinta de las Meditaciones cartesianas, por ejemplo, aunque el mismo título de las Ideas es indicación de los niveles descriptivo y crítico de la filosofía de Husserl). Bien puede objetarse que esta crítica fenomenológica no es todavía una reflexión antropológica, la fenomenología de la fenomenología, o la teoría trascendental del método no es una antropología filosófica, pero sí lo es el esclarecimiento de la razón filosófica como la meta más elevada de una humanidad que tiende a justificar filosófica o racionalmente el sentido de su propia vida.

\section{AUtOTRASCENDENCIA Y TRASCENDENTALIDAD DE LO HUMANO}

La epojé, esto que podríamos llamar también la sofisticación histórica (el refinamiento pues) del primer acto filosófico, esta detención de los intereses inmediatos y del correr de la vida para hacer filosofía, abre, en sí misma en cuanto acto de una novedosa índole, una nueva dimensión de lo humano que rebasa el plano natural de la reflexión y representa una reforma de la praxis desde su más lejano arcaísmo: la ignorancia socrática, el asombro del origen de la filosofía, la duda cartesiana.

La soledad del filósofo que contraviene la espontaneidad comunitaria del hombre no es, sin embargo, una «compensación» ${ }^{10}$ respecto de la idea de su ser fallido, es, sobre todo, la posibilidad de su auto-trascendencia. La afirmación del hombre en su inmediatez entraña en sí misma la posibilidad de su quiebre. Esta es la brecha que abre la filosofía en su innecesariedad material. La fenomenología, profundiza este quiebre en el curso de la filosofía. La epojé es menos el vaciamiento de la vida que la exposición de esta posibilidad huma-

\footnotetext{
vez de haber entrado aquí en la problemática ulterior y última de la fenomenología -la de la crítica de sí misma con vistas a la determinación del alcance y los límites, pero también de los modos de la apodicticidad-hemos dado la preferencia a la traza del bosquejo de la inmensa problemática de la fenomenología primera, en la cual (aunque ella misma está afectada aún, a su modo, de una ingenuidad: la ingenuidad apodíctica) se halla el gran rendimiento de la fenomenología, absolutamente privativo suyo, en tanto que configuración nueva y superior de la ciencia." Husserl, E., Meditaciones cartesianas, F.C.E., México, 2005, § 63, p. 205. Trad. José Gaos y Miguel García Baró.

${ }^{10} \mathrm{Me}$ refiero al concepto que Odo Marquard aporta a través de su filosofía de la compensación. La «compensación» sería en este sentido una huida humana de su propia falta. Un tratar de escapar de sus propia limitaciones expuestas -sobre todo- por la filosofía moderna y su idea de progreso. "Esto implica a su vez que el concepto de compensación, que entra en acción en la antropología filosófica de nuestro siglo, no procede originariamente del ámbito del psicoanálisis (...) más bien lo tomaron de un debate sobre la compensación en la fisiología cerebral que tuvo lugar en torno al cambio de siglo y que resumió Anton en 1905 en su conferencia de Jena «Über den Weiderersatz der Funktion bei Erkrankungen des Gehirns» [Sobre la sustitución de la función en enfermedades cerebrales]." Marquard, O., Filosofía de la compensación, Paidós, Barcelona, 2000, p. 20.
} 
na de autotrascendencia y, con ello, la radical superación de la antropología del hombre en falta. Esto, desde luego, nos obliga a asumir que quien lleva a cabo la epojé es el yo humano. En cierta medida, al menos para los fines primeros, los descriptivos, esto puede resultar irrelevante, e incluso, como advierte Husserl, se puede correr el riesgo de suponer que el yo puro es sólo una abstracción de lo humano ${ }^{11}$. Sin embargo, en la medida en que nos preguntamos por el sentido de la identidad del yo puro, tal como Husserl lo hizo sistemáticamente, llegamos -en el segundo libro de las Ideen ${ }^{12}$ a esa misma identidad yoica encarnada en una corporalidad sintiente, un elemental estrato de toda yoidad. Se trata, éste, de un profundo enriquecimiento del yo puro que tiene sendas repercusiones en la idea del yo personal humano.

La autocaptación de la que el yo personal o el yo trascendental se da bajo ciertas «variaciones» o posibilidades correlativas de una corporalidad sintiente. Tenemos pues, por un lado, esta posibilidad de la auto-trascendencia humana a través de la filosofía, por otro, el develamiento de una dimensión trascendental (es decir constituyente) de la humanidad a través de su corporalidad, por ambas vías hemos traspasado o superado la idea del hombre en falta rechazada explícitamente por Husserl. Y nos queda de frente el problema de la identidad corporal del yo personal humano y el yo puro o la subjetividad trascendental.

En el segundo libro de las Ideas aparecen los análisis de la corporalidad en relación con las descripciones del yo puro y la vida anímica. Se presenta aquí una franca dificultad para la consideración del cuerpo como pura trascendencia según el motivo que nos llevó a su desconexión. Y es que la cualidad por la que aparece es la de su ser sintiente y portador de sensaciones localizadas. ${ }^{13} \mathrm{El}$ cuerpo se distingue por eso de las cosas, porque las cosas sentidas y como tales objetivadas no son cosas a su vez sintientes. Las consideraciones constitutivas del yo hombre como naturaleza y el yo hombre como espíritu tienen como tema primero y fundamental las ubiestesias en tanto cualidad central (y centra-

\footnotetext{
${ }^{11}$ Cfr. Husserl, E., Ideas I, § 61, p.p. 215-218.

12 "Encontraremos ocasión, por lo demás, de dedicar a las difíciles cuestiones del yo puro, y a hacer más segura la posición que hemos tomado aquí provisionalmente, un capítulo propio en el libro segundo de este escrito." Husserl, E., Ideas I, § 57, p. 209.

13 "El cuerpo, por ende, se constituye primigeniamente de manera doble: por un lado es cosa física, materia, tiene su extensión, a lacual ingresan sus propiedades reales (...); por otro lado, encuentro en él, y siento 'en' él y 'dentro' de él: el calor en el dorso de la mano, el frío en los pies." Husserl, E., Ideas relativas a una fenomenología pura y una filosofía fenomenológica. Libro segundo: Investigaciones fenomenológicas sobre la constitución, F.C.E.-UNAM, México, 2005, § 36, p. 184. Trad. Antonio Zirión Quijano. (En adelante, Ideas II)
} 
lizadora) de la corporalidad. La autocaptación reflexiva de la persona humana es lo que constituye la realidad que fenomenológicamente llamamos espíritu, unidad corporal-anímica que se presenta en la autocaptación:

El yo espiritual -nos dice Husserl- depende del alma, y el alma del cuerpo; está, pues, naturalmente condicionado; pero no por ello está en una relación de causalidad con la naturaleza. tiene un subsuelo que es condicionalmente dependiente; como espíritu, tiene un alma, un complejo de disposiciones naturales que, como tales, están condicionadas por la naturaleza física y son dependientes de ella. ${ }^{14}$

El concepto de «yo hombre» está fundado en la autocaptación. La persona humana es siempre una posibilidad de la autorreflexión que capta o se capta como una mismidad unitaria de lo corporal-anímico. El hombre es unidad vital y realidad anímica intersubjetiva. Es intersubjetiva porque desde siempre mi cuerpo es también cuerpo expuesto a los otros, que completan mi vista de mí mismo. Así, la experiencia de los otros, en la llamada con más o menos rigor empatía, forma de darse del otro, es una dación intracomprensiva de lo anímico en la que el cuerpo juega el papel expresivo de lo espiritual, ${ }^{15}$ y es también experiencia de mi mismo, ya que completa mi autoexperiencia. Pero en esa misma medida el otro dado en la empatía es un otro trascendental porque me constituye, y a través de él el mundo es mundo objetivo.

Pero para terminar tengo que resolver una dificultad adicional que surge en el horizonte, porque estoy tratando de probar que el concepto de humano derivado del método fenomenológico (la epojé), es una superación de la idea del hombre en falta, pues éste descubre la dimensión trascendental de su ser, lo que permitiría elaborar una antropología filosófica centrada en ese sentido trascendental de la vida humana, solucionando así también la carencia antropológica de la fenomenología. Pero llegados aquí, la dificultad proviene del hecho de la posición de los animales que por ser sentientes también ellos son subjetividades trascendentales. Si el otro es puesto como subjetividad trascendental por su cuerpo expresivo ante todo de su vida sensible, también los animales, en los diferentes grados, serán subjetividades trascendentales ${ }^{16}$ porque también ellos

\footnotetext{
${ }^{14}$ Husserl, E., Ideas II, § 62, p. 330.

15 "El cuerpo no es solamente para mí, en tanto que es mediador de mis percepciones, de los efectos que produzco en el mundo de las cosas; aprehendido por el otro, alcanza su significado, un significado espiritual, en tanto que expresa lo espiritual (no sólo denuncia la sensibilidad)." Husserl, E. Ideas II, § 62 , p. 331.

16 "Si yo pongo en mi como hombre en el mundo un yo puro, es decir, veo que el yo-hombre es yo trascendental objetivado e igualmente lo hago en el caso de los otros, no puedo actual arbitrariamente al llegar al caso de los animales; si también ellos tienen una vida anímica que sea igualmente punto de referencia de la unidad del mundo, esa vida anímica que sea igualmente punto de referencia de la uni-
} 
ordenan y generan sus mundos de la vida (dejaremos el mundo vegetal porque su vida no es sintiente). Pero entonces cabría hablar en relación con ellos de una posibilidad de un estudio filosófico equivalente a la antropología filosófica. Quiero decir que con lo dicho antes, no he mostrado aún el sentido trascendental del humano que legitime una antropología filosófica.

Por tanto, con haber mostrado una identidad corporal entre el yo humano y el yo trascendental, esto es, si bien la subjetividad trascendental tiene un cuerpo, no es este todavía un cuerpo humano, no al menos de un modo necesario. Por ser los animales también subjetividades trascendentales, el análisis de la vida anímica nos ha dado por resultado la descripción de ciertas estructuras universales de una actividad constituyente, en la que tendríamos que ubicar, aunque quizás escalonadamente -en referencia a nuestra propia estructura fisiológica- a los animales (dejemos de lado las plantas, porque de estas últimas nada podamos saber debido a las diferencias estructurales inmensas que guardan con nuestra propia corporalidad). Un estudio de las estructuras constituyentes de la vida animal sólo podría partir, de hecho, de las condiciones de mi propio cuerpo, a partir de mi propia experiencia puedo intentar acercarme a los horizontes experienciales de subjetividades que perciben a través de una organicidad variable. Tal posibilidad implica el reconocimiento de la subjetividad trascendental animal o de que toda conciencia es conciencia trascendental, constituyente de un mundo que desborda el mundo físico y que es el mundo de la vida, que primariamente es de la vida animal. Este reconocimiento se da ya sobre la base de la encarnación de la subjetividad trascendental en su cuerpo.

Una vez expuesta la dificultad de que tener un cuerpo sentiente es encarnar la subjetividad, lo que llevaría a difuminar el sentido trascendental de lo humano, al tenerlo también el resto de los animales, por lo que afirmar que el yo puro tiene un cuerpo, no equivale a afirmar que la subjetividad trascendental tiene que ser el hombre. Sin embargo, las diferencias estructurales entre la conciencia trascendental humana y la subjetividad trascendental animal no se refieren sólo a la estructura fisiológica de los animales y la del hombre. Se trata de cierta capacidad racional y de autotrascendencia a través de la filosofía lo que de entrada impediría que nos planteáramos sobre un mismo plano la idea

dad del mundo, esa vida anímica tiene que tener un sentido trascendental, es decir, es constituyente." San Martín, J., Para una filosofía de Europa. Ensayos de fenomenología de la Historia, Biblioteca Nueva UNED, Madrid, 2007, p. 49. 
de una antropología filosófica y la de una -por ejemplo- «elefantología filosófica» ${ }^{17}$. El análisis de las estructuras trascendentales de la animalidad, como análisis de la constitución, pueden bien quedar enmarcados en los límites de una ontología regional, pero no así la descripción de las estructuras trascendentales de la subjetividad humana. Pues el sentido de lo humano fenomenológicamente develable- a través de la crítica filosófica del sentido de su ser racional (meta que coincide con la de la filosofía fenomenológica) desborda los límites de toda parcelación ontológica. Esta capacidad crítica de una racionalidad potenciada es racionalidad que desborda los límites de la animalidad en sentido fáctico o biológico, haciendo visibles las estructuras trascendentales de la vida anímica autorreflexiva. La idea de un hombre que es capaz de dar razón de su razón, de autojustificarse radicalmente, la idea de un sujeto que puede hacerse cargo de sí mismo, por sí mismo, al menos una vez en la vida, es la idea fenomenológica de lo humano. Esto sólo quiere decir que la crítica fenomenológica reforma la idea del hombre a través del ideario del hombre que filosofa.

La idea trascendental de lo humano es necesaria frente a la antropología del hombre en falta, que es decir, frente a la crisis de la humanidad (que filosofa). El origen de la crisis no está fuera de la filosofía. La humanidad en crisis es un síntoma de la crisis del pensamiento, del reciente olvido de la filosofía de su vocación, de sus verdaderos problemas, los problemas humanos.

Hay un punto en la historia del pensamiento filosófico en el que la filosofía exige de sí misma un rendir cuentas de su sentido ¿Por qué y para qué filosofar? ¿Por qué y para qué la fenomenología? ¿Para qué quiero claridad? Para poder vivir sin contradicciones. La autenticidad de esta vida no es extrínseca al acto filosófico, sino que le corresponde originariamente. ¿Qué queda del hombre sin la dimensión trascendental de su ser? Qué queda del hombre sin la filo-

\footnotetext{
${ }^{17}$ Quisiera citar al respecto, algunas líneas de Antonio Zirión escritas para el Prologo del primer volumen sobre Antropología y fenomenología: "Aquí está en juego, por tanto, la esencia misma de la filosofía, de modo que el debate relativo a la posibilidad de una antropología filosófica es en cierta forma doble: por un lado está la cuestión misma de la filosofía, y por otro lado, pero en íntima conexión con el modo como esta cuestión se resuelva o se asuma, está la cuestión del sentido de una antropología filosófica, o sea, de una filosofía del hombre. ¿Por qué es posible, puede preguntarse, una filosofía del hombre y no, digamos, una filosofía de los mamíferos en general, o de los leones o de los platelmintos? De modo que en el tema de la definición o caracterización de la antropología filosófica no está sólo en cuestión la esencia de la filosofía, sino también, y sobre todo, la de la esencia misma del hombre. Esto se puede apreciar también por otro aspecto de esta problemática." Zirión, Q., A., en su "Prologo" a Antropología y fenomenología I. Reflexiones sobre historia y cultura, ENAH, (varios autores) en prensa, México, 2014, p. 4.
} 
sofía, sólo un ser necesitado, queda el hombre, como con claridad lo vería Eduardo Nicol, a la intemperie.

\section{BIBLIOGRAFÍA CITADA}

Blumenberg, H., Descripción del ser humano, F.C.E., Buenos Aires, 2011. Trad. Griselda Mársico.

Husserl, E. Ideas para una fenomenología pura y una filosofía fenomenológica. Libro primero: Introducción general a la fenomenología pura, F.C.E., México, 2013. Trad. José Gaos. Edición y refundición integral, Antonio Zirión Quijano.

- Investigaciones lógicas, 1., Alianza, Madrid, 2006. Trad. Manuel García Morente y José Gaos

- Ideas relativas a una fenomenología pura y una filosofía fenomenológica. Libro segundo: Investigaciones fenomenológicas sobre la constitución, F.C.E.-UNAM, México. Trad. Antonio Zirión Quijano.

- Meditaciones cartesianas, F.C.E., México, 2005. Trad. José Gaos y Miguel García Baró.

- Problemas fundamentales de la fenomenología, Alianza Universidad, Madrid, 1994. Trad. Javier San Martín y César Moreno.

Marquard, O., Filosofía de la compensación, Paidós, Barcelona, 2000.

Nicol, E., La reforma de la filosofía, F.C.E., México, 1989.

San Martín, J., Fenomenología y antropología, Lectour-UNED, Buenos Aires/Madrid, 2005.

- Para una filosofía de Europa. Ensayos de fenomenología de la Historia, Biblioteca Nueva UNED, Madrid, 2007.

Zirión, Q., A., Antropología y fenomenología I. Reflexiones sobre historia y cultura, ENAH (en prensa). 